\title{
PUFA levels reduced in schizophrenia patients
}

Published online: 18 October 2012

(C) Springer Healthcare 2012

medwireNews: Patients with schizophrenia exhibit reduced red blood cell levels of certain polyunsaturated fatty acids (PUFAs), results from a systematic review and meta-analysis show.

Analysis of results from 18 studies indicate that schizophrenia patients have reduced erythrocyte cell-membrane levels of the PUFAs docosapentaenoic acid (DPA), docosahexaenoic acid (DHA), linoleic acid (LA), and arachidonic acid (AA) compared with mentally healthy individuals (controls).

However, Wendela Hoen (Mentrum Institute for Mental Health, Amsterdam, the Netherlands) and colleagues warn that "given the large heterogeneity in results, these conclusions should be interpreted cautiously.”

In total, 642 schizophrenia patients and 574 controls participated in the studies, which were published between 1986 and 2011. Of the schizophrenia patients, 169 were antipsychotic-naïve.

The team found that patients taking antipsychotic medication and antipsychotic-naïve patients had significantly decreased levels of DPA and DHA compared with controls.

Regarding LA levels, antipsychotic-treated patients showed significantly reduced levels compared with controls, whereas antipsychotic-naïve patients showed no such reductions.

Conversely, antipsychotic-naïve patients showed significant reductions in levels of AA compared with controls, whereas evidence for such reductions in antipsychotic-treated patients was "less convincing."

Hoen and team conclude in Psychiatry Research: "Taken together, there is suggestive evidence that decreased levels of DPA, DHA, and AA are associated with the schizophrenia syndrome, apart from a possible influence of antipsychotic medication."

They add: "Further research should focus on antipsychotic-naïve patients versus patients that use antipsychotics, with special attention for the type of antipsychotics used, the dosage, and the therapeutic effect of the medication.”

By Mark Cowen, Senior medwireNews Reporter

\section{Reference}

Psychiatry Res 2012; Advance online publication 